\title{
Low-dose x-ray phase contrast tomography: experimental setup, image reconstruction and applications in biomedicine
}

\author{
Charlotte K. Hagen, Anna Zamir, Paul C. Diemoz, Marco Endrizzi, Fiona Kennedy, Rolf H. Jager, and \\ Alessandro Olivo
}

\begin{abstract}
An unmet demand for high resolution tomographic imaging modalities providing enhanced soft tissue contrast exists in a number of biomedical disciplines. $X$-ray phase contrast imaging (XPCi) methods can provide a solution: contrast is driven by phase (refraction) effects rather than attenuation effects, the formers being much larger than the latters for weakly attenuating materials and energies typically used for biomedical imaging. However, the majority of the existing XPCi methods suffer from drawbacks affecting their implementation outside specialized facilities such as synchrotrons and therefore their applicability to biomedical research. The Edge Illumination (EI) XPCi method has the potential to overcome or at least mitigate most of these drawbacks. Its major strengths are its simple setup, compatibility with commercially available $x$-ray tubes and potential for low-dose imaging. EI XPCi has recently been implemented as a tomographic modality, and it was demonstrated that the method can provide quantitatively accurate volumetric images acquired with low entrance doses. This paper explains the experimental requirements for tomographic EI XPCi, outlines the image reconstruction process and discusses potential applications in biomedicine. As an example, first experimental images of an atherosclerotic plaque specimen are presented.
\end{abstract}

\section{INTRODUCTION}

$\mathbf{R}$ ADIOGRAPHIC imaging of soft biological tissues is considered difficult due to their similar attenuation properties. X-ray phase contrast imaging (XPCi) has been demonstrated to provide improved image contrast also when the $\mathrm{X}$ ray attenuation are similar, as the main contrast mechanism is the phase shift that $\mathrm{x}$-rays suffer while they pass through a sample. This effect can be much larger than attenuation effects for photon energies used for biomedical applications [1]. Most XPCi methods have originally been developed using the spatially and temporally coherent beams of synchrotron radiation facilities [2]-[4]. Over the last decade, however, the translation of XPCi into standard research laboratories has been heavily investigated, and XPCi methods were developed that are compatible with $\mathrm{x}$-ray tubes with extended focal

Manuscript received November 14, 2014. This work was supported by the UK Engineering and Physical Sciences Research Council (Grant Nos. EP/L001381/1 and EP/I021884/1). P. C. Diemoz and M. Endrizzi are supported by Marie Curie Career Integration Grant Nos. PCIG12-GA-2012333990 and PCIG12-GA-2012-334056 within the Seventh Framework of the European Union.

C. K. Hagen, A. Zamir, P. C. Diemoz, M. Endrizzi and A. Olivo are with the Department of Medical Physics and Biomedical Engineering, University College London, Malet Place, Gower Street, London WC1E 6BT, UK (e-mail: charlotte.hagen.10@ucl.ac.uk).

F. Kennedy and R. H. Jager are with the Institute of Neurology, University College London, Queen Square, London WC1N 3BG, UK. spots and broad energy spectra [5], [6]. This has been an extremely important step as it enables a widespread diffusion of XPCi into various biomedical disciplines. Another recent advancement of XPCi has been its combination with the principles of computed tomography (CT), enabling volumetric imaging [7]-[13].

This paper focuses on the tomographic implementation of the edge illumination (EI) XPCi method. EI XPCi is compatible with both synchrotron radiation and laboratory-based $\mathrm{x}$-ray tubes. Moreover, it is based on a simple experimental setup, highly sensitive to phase effects, relatively unaffected by environmental vibrations and dose efficient [14]-[20]. Hence, it is well suited to biomedical imaging applications. After having published recently on the quantitative accuracy and the low-dose capabilities of tomographic EI XPCi [11]-[13], we have now started to investigate biomedical applications that can benefit from the method, including the imaging of atherosclerotic plaques in carotid arteries, which is considered challenging with conventional, non-contrast agent assisted radiographic techniques. This paper explains the experimental requirements for tomographic EI XPCi acquisitions, outlines the CT reconstruction process and discusses potential applications of the method to biomedical specimens that are currently under investigation by our group. Moreover, the first volumetric images of an atherosclerotic plaque specimen that were acquired with a tomographic EI XPCi scanner implemented with standard, off-the-shelf $\mathrm{x}$-ray equipment are presented. Measurements of the entrance dose for the scans were taken and the results are presented.

\section{MAterials And MetThods}

\section{A. Experimental setup}

The setup of EI XPCi in its original form involves a slit positioned in front of the object, typically collimating the beam down to $10-20 \mu \mathrm{m}$, and a second slit in front of the detector, positioned so as to stop half of the (then laminar) beam, while allowing the remaining half through [Fig. 1(a)]. In this "edge illumination" configuration sensitivity to refraction in addition to attenuation is created: $\mathrm{x}$-rays which are deviated towards (away from) the uncovered detector area cause an increased (a decreased) measured intensity. An image can be obtained by scanning the object vertically through the setup. This requirement can lead to long acquisition times when $\mathrm{x}$ ray flux is limited, e.g. when x-ray tubes are used instead of 
synchrotron radiation. To solve this problem, an extended EI XPCi setup has been developed in which an apertured mask ("sample mask") is used instead of a slit, splitting the beam into an array of laminar beams [Fig. 1(b)]. A second apertured mask ("detector mask"), creating insensitive areas between the pixel rows, replaces the slit in front of the detector. This extended setup allows obtaining an image in a single shot, but it should be noted that the spatial sampling rate is determined by the distance between the laminar beams. A higher sampling rate can be achieved by scanning the sample through the beams by the distance that separates them. Tthe relatively large focal spot of $\mathrm{x}$-ray tubes and the resulting beam incoherence are not a problem, provided that the laminar beams are physically separated.

Any given EI XPCi setup is characterized by the so-called illumination curve (in the following denoted by $C$ ), which can be measured in the absence of an object, by scanning the sample mask relative to the detector mask in the direction orhogonal to the mask trenches (i.e. along $x$ ). The illumination curve is a monotonically increasing function until the apertures in sample and detector mask are aligned with each other; here, the curve take its maximum (100\% illumination). Afterwards, the curve falls monotonically until the apertures in the sample mask are alinged with the absorbing parts of the detector mask; here, the curve takes on its minimum ( $0 \%$ illumination).

An image acquired with EI XPCi shows a combination of refraction (high frequency edge contrast) and attenuation information (low frequency area contrast). It should be noted that refraction is sensed in one direction only (in the direction orthogonal to the mask trenches). The possibility to sense refraction in two dimensions has been demonstrated previously [25] and is currently under further development. The unidirectional refraction contrast can be inverted by repositioning the sample mask such that the laminar beams illuminate the opposing edges created by the detector mask: deviated $\mathrm{x}$ rays that previously caused an increased measured intensity now cause a decreased intensity and vice versa. A dedicated algorithm, taking as input two images acquired under opposing edge illumination conditions (in the following denoted by $I_{1}$ and $I_{2}$ ), enables the retrieval of separate refraction and attenuation images [21]. As will be explained in Section II.B., from a mathematical point of view, this algorithm provides the basis for EI XPCi tomography. Experimentally, tomographic acquisitions with an extended setup as shown in Fig. 1(b) require rotating the object over a range of at least 180 degrees and acquiring images at multiple view angles. The axis of rotation is aligned with the y-axis.

\section{B. Image reconstruction}

In $\mathrm{x}$-ray imaging, the refracting and attenuating properties of a material are typically described by the complex refractive index, given by:

$$
n(E)=1-\delta(E)+i \beta(E),
$$

(a)

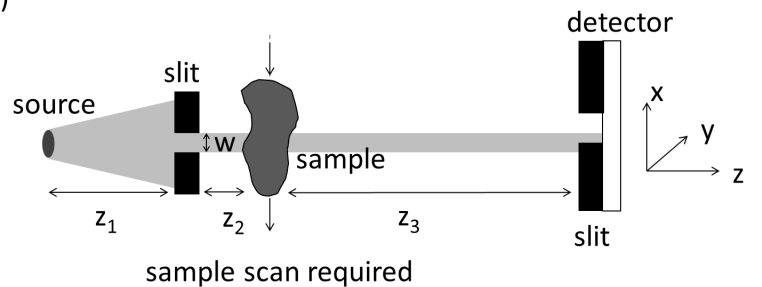

(b)

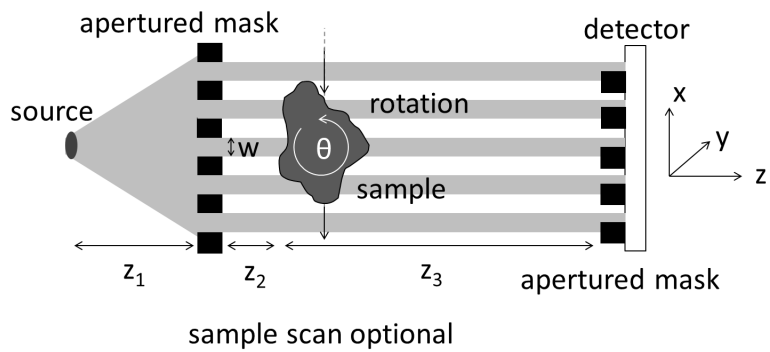

Fig. 1. Single slit (a) and extended (b) implementations of (tomographic) edge illumination $\mathrm{x}$-ray phase contrast imaging.

where $E$ is the photon energy. The phase shift $(\Phi)$ and attenuation $(M)$ caused by an object are given by:

$$
\begin{aligned}
\Phi(x, y) & =k \int_{\mathcal{O}} \delta(x, y, z) d z \\
M(x, y) & =2 k \int_{\mathcal{O}} \beta(x, y, z) d z,
\end{aligned}
$$

where the integration is performed along the $\mathrm{x}$-ray path ( $\mathrm{z}$ axis) and over the extent of the object, and $k$ is the wave number. The refraction angle $(\alpha)$ and the beam transmission $(T)$ can be calculated as:

$$
\begin{aligned}
& \alpha(x, y)=\frac{1}{k} \Phi^{\prime}(x, y) \\
& T(x, y)=e^{-M(x, y)},
\end{aligned}
$$

where $\Phi^{\prime}$ denotes the derivative of the phase shift along the direction of phase sensitivity (i.e. along the x-axis in Fig. 1).

Under the assumption that $\alpha$ is constant within each aperture in the sample mask and considering that $\alpha$ is very small (microradians), refraction corresponds to a shift of the beam by a distance of $z_{3} \alpha$ on the detector plane. Neglecting ultrasmall angle scattering, a simple model to describe the intensity on the detector in dependence of the refraction and attenuation caused by an object is given by [15]:

$$
\begin{aligned}
& I_{1}(x, y)=I_{0} \cdot T(x, y) \cdot C\left(x_{1}+z_{3} \alpha(x, y)\right) \\
& I_{2}(x, y)=I_{0} \cdot T(x, y) \cdot C\left(x_{2}+z_{3} \alpha(x, y)\right) .
\end{aligned}
$$

$I_{1}$ and $I_{2}$ are the images acquired under opposing illumination conditions, and $x_{1}$ and $x_{2}$ are the corresponding positions of the sample mask. Considering that $z_{3}$ is known and $I_{0}$ and $C(x)$ can be measured independently before the image acquisition, (6) and (7) allow extracting $M$ and $\alpha$. The precise formulas are derived and provided in reference [21]. If $I_{1}$ and 
$I_{2}$ are acquired in a tomographic fashion, i.e. by rotating the object and acquring $I_{1}$ and $I_{2}$ at every angle, these formulas allow extracting sinograms of the attenuation and the refraction angle, given by:

$$
\begin{aligned}
S_{M}(x, y ; \theta) & =2 k \int_{\ell(x, y ; \theta)} \beta\left(x^{\prime}, y, z^{\prime}\right) d z \\
S_{\alpha}(x, y ; \theta) & =\frac{\partial}{\partial x} \int_{\ell(x, y ; \theta)} \delta\left(x^{\prime}, y, z^{\prime}\right) d z .
\end{aligned}
$$

For a given rotation angle $\theta$, the line $\ell(x, y ; \theta)$ describes the path of x-rays reaching the detector at location $(x, y)$, and $\left(x^{\prime}, y, z^{\prime}\right)$ describes the coordinate system of the sample that rotates around the $y$-axis. From the sinograms described by (8) and (9), tomograhic maps of $k \beta$ and $\delta$ can be reconstructed with standard CT reconstruction methods, e.g. filtered back projection (FBP) or the algebraic reconstruction technique (ART). Please note that the reconstruction of $\delta$ requires an additional one-dimensional integration to eliminate the derivative in Eq. 9. Alternatively, the integration can be implicitely performed in Fourier space by using a specialized filter function with the FBP [23]. When imaging is performed with a monochromatic beam, a map of $\beta$ can be obtained via a division of the $k \beta$-map by the wave number. This is not possible when a polychromatic beam is used; in this case, the reconstructed $k \beta$ and $\delta$-maps refer to effective energies [12], [24].

\section{RESUlTS AND DISCUSSION}

\section{A. Applications in Biomedicine}

Potential biomedical applications of EI XPCi include all fields of research where conventional radiography provides insufficient soft tissue contrast. Among these are, for example, mammography, the imaging of articular cartilage for improved diagnosis of osteoarthritis, prostate cancer imaging and the imaging of plaques for improved detection of atherosclerosis. Small animal imaging is another potential application that can involve all of the above. The named areas were, or are currently, explored both with planar and tomographic EI XPCi. The benefit of the method for mammography was investigated with a planar, laboratory-based setup. The images, which were evaluated by radiologists and compared to conventional mammographic images, showed improved contrast and a higher detection rate for micro-calcifications [20]. Moreover, it was demonstrated that planar, laboratory-based EI XPCi can visualize $100 \mu \mathrm{m}$ thin cartilage layers in dissected rat joints, as well as detect small defects, which could be early indicators for osteoarthritis [22]. The benefits of tomographic EI XPCi for prostate cancer, small animal and atherosclerotic plaque imaging are currently under investigation by our group.

\section{B. Imaging of an atherosclerotic plaque sample}

As an example of a potential biomedical application of tomographic EI XPCi, we discuss here the imaging of an atherosclerotic human carotid artery plaque specimen. Plaques are lesions forming on the inside of arterial walls, and have various components (e.g. collagen, lipids, calcifications and intraplaque haemorrhage). Vulnerable plaques, i.e. those most likely to rupture and cause conditions like strokes and heart attacks, are typically characterized by lipid-rich necrotic cores contoured by thin fibrous caps. The detection of atherosclerotic plaques and their classification as vulnerable or nonvulnerable, based on the discrimination of the different components, is difficult with conventional computed tomography due to poor soft-tissue contrast. The aim of this preliminary investigation was to determine whether it is possible to discriminate different components within the plaque using tomographic EI XPCi. This would be an important step towards assessing the likelihood of a plaque to cause serious problems, thus facilitating diagnostic decisions.

The imaged specimen originates from a stenosis in a human carotid artery that was removed from a patient via carotid endarterectomy. The imaging of the specimen was approved by the local ethics committee. The plaque was fixed in a formalin solution, and, for the image acquisition, was placed inside a plastic cylinder of approximately $5 \mathrm{~mm}$ diameter. The imaging was performed with an extended EI XPCi setup implemented with the commercially available Rigaku MicroMax $007 \mathrm{HF}$ rotating anode (molybdenum) $\mathrm{x}$-ray tube (Rigaku Corporation, Japan), operated at $35 \mathrm{kVp}$ and $25 \mathrm{~mA}$. The broad energy spectrum was filtered with a $30 \mu \mathrm{m}$ thick molybdenum sheet. The size of the source's focal spot was measured to be approximately $70 \mu \mathrm{m}$. As a detector, the Anrad SMAM aSe direct conversion flat panel (Analogic Canada Corporation, Canada) with a pixel size of $85 \mu \mathrm{m}$ was used. The distances involved in the setup were approximately $1.6 \mathrm{~m}\left(z_{1}\right), 5 \mathrm{~cm}$ $\left(z_{2}\right)$ and $0.35 \mathrm{~m}\left(z_{3}\right)$, respectively. Both masks were fabricated by electroplating gold strips onto a graphite substrate (Creatv Microtech Inc., Potomac, MD). The masks' periods were 66.8 $\mu \mathrm{m}$ (sample mask) and $83.5 \mu \mathrm{m}$ (detector mask), with open fractions of $18 \%(12 \mu \mathrm{m})$ and $24 \%(20 \mu \mathrm{m})$, respectively.

The tomographic rotation was carried out over a total angular range of 180 degrees with a 1 degree step. At each angle, two images were acquired under opposing edge illumination configurations, with the sample mask positioned such that $50 \%$ of each beam fell onto the uncovered pixel area. For each image, the sample was exposed to $\mathrm{x}$-rays for 3 s. The entrance dose (air kerma) was measured by means of thermoluminescence devices (TLD-100H), placed at the position of the object. The TLDs had been calibrated in air kerma against a reference ion chamber. The measurements were repeated with an independent ion chamber (Keithley $35050 \mathrm{~A}$ ) and the same entrance doses were obtained within approximately $10 \%$ accuracy. The data processing involved the extraction of attenuation and refraction angle sinograms [21]. FBP was employed to reconstruct tomographic maps of $k \beta$ and $\delta$. In the latter case, the Hilbert filter was used in combination with FBP [23].

Figures 2 and 3 show the reconstructed EI XPCi images of the plaque. Transverse, coronal and sagittal cross sections of the reconstructed $\delta$-map can be seen in Fig. 2. While the transverse cross section exposes the lumen, in the coronal and sagittal cross sections the narrowing and, partially, the complete obstruction of the lumen (stenosis) can be clearly appreciated. These reveal a slight difference in the grey values 


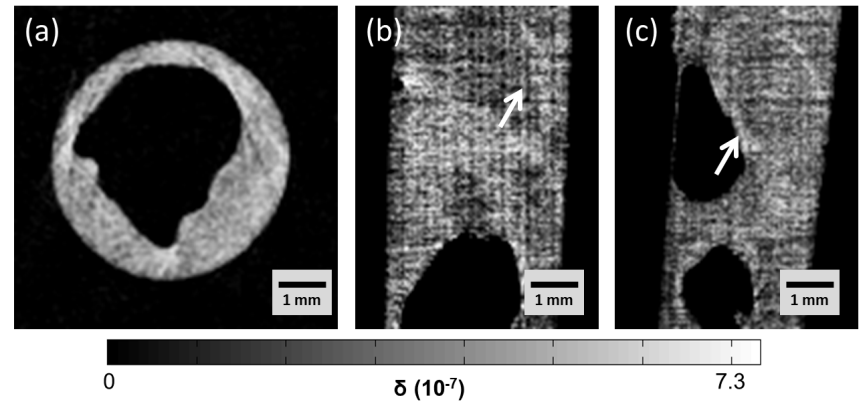

Fig. 2. Reconstructed $\delta$-maps of the imaged atherosclerotic plaque sample: transverse (a), coronal (b) and sagittal (c) cross sections.

within the plaque, indicating different tissue components. Figures 3(a)-(c) show volume renderings of the reconstructed $\delta$-map. Figure 3(a) and 3(b), showing a top view and a cropped side view respectively, render the data in grey scale, and Fig. 3(c) renders the data in a false color scale. In Fig. 3(a), the narrowing and ultimately complete closure of the lumen (stenosis) are apparent. The non-homogeneous plaque composition can be seen in Fig. 3(b), and is even more apparent when displayed in false colors in Fig. 3(c). For comparison, Fig. 3(d) shows a volume rendering of the reconstructed $k \beta$-map, rendered in the same false color scale as the $\delta$-map in Fig. 3(c). It is easily appreciated that the contrast-to-noise ratio in the $k \beta$ map is lower than in the $\delta$-map, to an extent that no tissueinhomogeneouity can be detected. The measured total entrance dose for the scan leading to the images in Figs. 2 and Fig. 3 was below $50 \mathrm{mGy}$, a value about a tenth of the limits imposed by e.g. in vivo small animal imaging. The dose efficiency of EI XPCi has been observed previously [20] and can be attributed to the sample mask being located upstream of the sample and the masks substrate being made of low-attenuating graphite.

\section{CONCLUSiON}

An introduction to phase contrast tomography using the EI XPCi method was provided, including a description of the experimental setup and image reconstruction procedure. An important feature of tomographic EI XPCi is that it allows reconstructing three-dimensional maps of $\delta$ and $\beta$, which are the two components of the complex refractive index $n$ [Eq. 1]. A number of applications in the biomedical field that can potentially benefit from tomographic EI XPCi are currently under investigation. These include the imaging of atherosclerotic plaques for improved classification of atherosclerosis. As an example, tomographic EI XPCi images of a carotid artery plaque sample were shown. Although these are preliminary results, they demonstrate the superiority of phase over attenuation imaging for this type of samples. In particular, the results indicate that tomographic EI XPCi provides the necessary soft tissue contrast to discriminate different tissue types in an atherosclerotic plaque. The preliminary images motivate further investigation involving the imaging of a range of specimen with different pathologies and histological confirmation of the results.
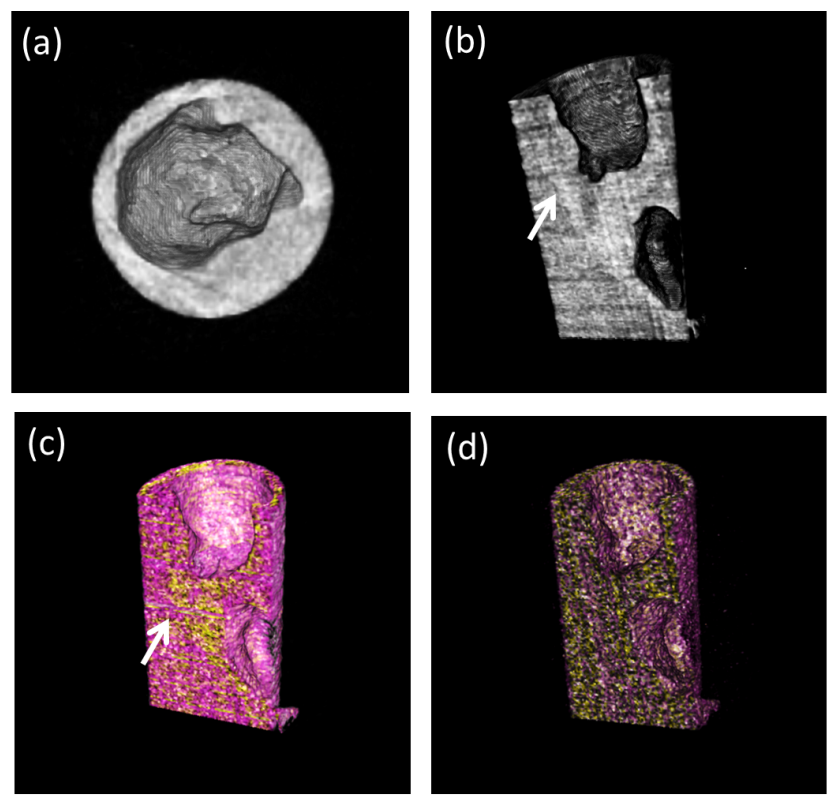

Fig. 3. Volumetric representations of the imaged atherosclerotic plaque sample, created with the open source software ImageJ: (a), (b) grey scale rendering of $\delta$-map, (c) false color rendering of $\delta$-map and (d) false color rendering of $k \beta$-map.

\section{REFERENCES}

[1] R. Lewis, C. Hall, A. Hufton, S. Evans, R. Menk, F. Arfelli, L. Rigon, G. Tromba, D. Dance, I. Ellis, A. Evans, E. Jacobs, S Pinder and K. Rogers, "X-ray refraction effects: application to the imaging of biological tissues," The British Journal of Radiology 76, 301-308 (2003).

[2] V.N. Ingal and A. Beliaevskaya, "X-ray phase-wave topography observation of the phase contrast from a non-crystalline object," Journal of Physics D: Applied Physics 28, 2314-2317 (1995).

[3] A. Snigirev, I. Snigireva, V. Krohn, S. Kuznetsov and I. Schelokov, "On the possibilities of X-ray phase contrast microimaging by coherent high energy synchrotron radiation," Review of Scientific Intruments 66, 5486-5492 (1995).

[4] A. Momose, S. Kawamoto, I. Koyama, Y. Hamaishi, K. Takai and Y. Suzuki, "Demonstration of X-ray Talbot interferometry," Japanese Journal of Applied Physics 42(Part2, No. 7B), L866L868 (2003).

[5] F. Pfeiffer, T. Weitkamp, O. Bunk and C. David, "Phase retrieval and differential phase contrast imaging with low-brilliance $\mathrm{x}$-ray sources," Nature Physics 2, 258-261 (2006).

[6] A. Olivo and R. Speller, "A coded-aperture technique allowing Xray phase contrast imaging with conventional sources," Applied Physics Letters 91, 074106 (2007).

[7] A. Momose, T. Takeda, Y. Itai and K. Hirano, "Phase-contrast Xray computed tomography for observing biological soft tissues," Nature Medicine 2(4), 473-475 (1996).

[8] P. Cloetens, W. Ludwig, J. Baruchel, D. Van Dyck, J. Van Landuyt, J.P. Guigay and M. Schlenker, "Holotomography: quantitative phase tomography with micrometer resolution using hard synchrotron radiation X-rays," Applied Physics Letters 75(19), 2912-2914 (1999).

[9] F. Dilmanian, Z. Zhong, B. Ren, X. Wu, L. Chapman, I. Orion and W. Thomlinson, "Computed tomography of $\mathrm{x}$-ray index of refraction using the diffraction enhanced imaging method," Physics in Medicine and Biology 45, 933-946 (2000).

[10] T. Weitkamp, A. Diaz, C. David, F. Pfeiffer, M. Stampanoni, P. Cloetens and E. Ziegler, "X-ray phase imaging with a grating interferometer," Optics Express 13(16), 6296-6304 (2005).

[11] C.K. Hagen, P.C. Diemoz, M. Endrizzi, L. Rigon, D. Droessi, F. Arfelli, F.C.M. Lopez, R. Longo and A. Olivo, "Theory and preliminary experimental verification of quantitative edge 
illumination x-ray phase contrast tomography," Optics Express 22(7), 7989-8000 (2014).

[12] C.K. Hagen, P.R.T. Munro, M. Endrizzi, P.C. Diemoz and A. Olivo, "Low-dose phase contrast tomography with conventional sources," Medical Physics 41(7) 070701 (2014).

[13] C.K. Hagen, P.C. Diemoz, M. Endrizzi and A. Olivo, "The effect of the spatial sampling rate on quantitative phase information extracted from planar and tomographic edge illumination x-ray phase contrast images," Journal of Physics D: Applied Physics 47455401 (2014).

[14] A. Olivo, F. Arfelli, G. Cantatore, R. Longo, R. Menk, S. Pani, M. Prest, P. Poporat, L. Rigon, G. Tromba, E. Vallazza and E. Castelli, "An innovative digital imaging set-up allowing a lowdose approach to phase contrast applications in the medical field," Medical Physics 28(8), 1610-1619 (2001).

[15] P.C. Diemoz, M. Endrizzi, C.E. Zapata, Z. Pesic, C. Rau, A. Bravin, I. Robinson and A. Olivo, "X-ray phase contrast imaging with nanoradian angular resolution," Physical Review Letters 110, 138105 (2013).

[16] P.C. Diemoz, C.K. Hagen, M. Endrizzi and A. Olivo, "Sensitivity of laboratory based implementations of edge illumination X-ray phase-contrast imaging," Applied Physics Letters 103, 244104 (2013).

[17] T.P. Millard, M. Endrizzi, K. Ignatyev, C.K. Hagen, P.R.T. Munro, R.D. Speller and A. Olivo, "Method for automatization of the alignment of a laboratory based x-ray phase contrast edge illumination system," Review of Scientific Instruments 84, 083702 (2013).

[18] M. Endrizzi, P.C. Diemoz, T.P. Millard, J.L. Jones, R.D. Speller, I.K. Robinson and A. Olivo, "Hard x-ray dark-field imaging with incoherent sample illumination," Applied Physics Letters 104, 024106 (2014).

[19] M. Endrizzi, F.A. Vittoria, P.C. Diemoz, R. Lorenzo, R.D. Speller, U.H. Wagner, C. Rau, I.K. Robinson and A. Olivo, "Phasecontrast microscopy at high X-ray energy with a laboratory setup," Optics Letters 39(11), 3332-5 (2014).

[20] A. Olivo, S. Gkoumas, M. Endrizzi, C.K. Hagen, M.B. Szafraniec, P.C. Diemoz, P.R.T. Munro, K. Ignatyev, B. Johnson, J.A. Horrocks, S.J. Vinnicombe, J.L. Jones and R.D. Speller, "Lowdose phase contrast mammography with conventional sources," Medical Physics 40(9), 090701 (2013).

[21] P.R.T. Munro, C.K. Hagen, M.B. Szafraniec and A. Olivo, "A simplified approach to quantitative coded aperture X-ray phase imaging," Optics Express 21(9), 11187-11201 (2013).

[22] M. Marenzana, C.K. Hagen, P. Das Neves Borges, M. Endrizzi, M.B. Szafraniec, K. Ignatyev and A. Olivo, "Visualization of small lesions in rat cartilage by means of laboratory-based $\mathrm{x}$ ray phase contrast imaging," Physics in Medicine and Biology 57(24), 8173-8184 (2012).

[23] Z. Huang, K. Kang, Z. Li, P. Zhu, Q. Yuan, W. Huang, J. Huang, D. Zhang and A. Yu, "Direct computed tomographic reconstruction for directional-derivative projections of computed tomography of diffraction enhanced imaging," Applied Physics Letters 89, 041124 (2006).

[24] P.R.T. Munro and A. Olivo, "X-ray phase contrast imaging with polychromatic sources and the concept of effective energy," Physical Review A 87, 053838 (2013).

[25] A. Olivo, S.E. Bohndiek, J.A. Griffiths, A. Konstantinidis and R.D. Speller, "A non-free-space propagation X-ray phase contrast imaging method sensitive to phase effects in two directions simultaneously," Applied Physics Letters 94, 044108 (2009). 